\title{
FREQÜÊNCIA DE AGLUTININAS PARA LEPTOSPIRA OBSERVADAS EM HABITANTES DE UBERABA, MINAS GERAIS
}

\author{
José Tavares-Neto, Jarbas Andrade, Ernesto Hofer, Guilherme \\ Ferreira de Oliveira e Abelardo Couto-Júnior
}

Mesmo nos países industrializados, o risco das doenças zoonóticas é crescente, devido inclusive ao contato com animais de estimação ${ }^{33}$. No Brasil, na região do Triângulo Mineiro, a agropecuária é desenvolvida, a população urbana tem contatos estreitos com o meio rural e a leptospirose em animais é conhecida $^{11}$. A literatura brasileira tem vários estudos soro-epidemiológicos semelhantes, em outras regiôes brasileiras, inclusive $\mathrm{em}$ humanos 356 .

Em Uberaba, cidade do Triângulo Mineiro, são raros os relatos de leptospirose - doença humana. No período de 1987-1990, por exemplo, somente um caso de leptospirose foi diagnosticado no Hospital-Escola da Faculdade de Medicina do Triângulo Mineiro. A freqüência de indivíduos na população com sorologia positiva para esta antropozoonose não é conhecida.

É conhecida, porém, a freqüência elevada das formas assintomáticas, oligossintomáticas e anictéricas, que ocorrem em todo o mundo ${ }^{1}$. Essas, muitas vezes, passam despercebidas ou ficam sem diagnóstico por falta de provas laboratoriais específicas, considerando que o diagnóstico clínico é difícil nestes casos.

Assim sendo, idealizamos o presente estudo seccional, pretendendo conhecer a freqüência da infecção em grupos de risco ou não, da população do município de Uberaba, estado de Minas Gerais. As variáveis pesquisadas estão na Tabela 1. A ocupacão foi classificada conforme o nível de especialização formal. O grau de contato com animais foi estimado através do somatório dos escores ordinais das seguintes variáveis: trabalho com animais; abate de animais; produção ou

Faculdade de Medicina da Universidade Federal da Bahia Salvador; BA; Fundacão Oswaldo Cruz, Rio de Janeiro, RJ e Faculdade de Medicina do Triângulo Mineiro, Uberaba, MG. Endereço para correspondência: José Tavares-Neto, Rua Marques de Caravelas 262/101, 40210-140 Salvador, BA. Recebido para publicação em 22/06/94. manipulação industrial ou caseira de produtos animais; consumo de carnes, derivados e leite nâo-pasteurizado; criação de animais de estimação e práticas de lazer com a participação de animais. Os graus de contato com animais com somatórios inferiores à mediana foram considerados menores.

A pesquisa de anticorpos circulantes específicos contra leptospirose foi feita pela técnica de micro-aglutinação, utilizando-se como antígeno culturas viáveis de 24 sorovars representativos dos sorogrupos que reconhecidamente mais incidem entre nós $s^{3}$, como: icterobaemorrbagiae (cepa 3294 e RGA), copenhageni, javanica, canicola, castellonis, pyrogenes, cynopteri, autumnalis, sentot, djasiman, australis, pomona, grippotyphosa, bebdomadis, wolffi, sejroe, saxkoebing, bataviae, tarassovi, panama, andamana, celledoni, shermani e patoc. O título mínimo considerado como positivo foi de $1: 200^{237}$

Entre os indivíduos pesquisados o grau de contato com animais foi maior entre os trabalhadores do frigorífico $(n=28)$, de aviários $(n=87)$, vaqueiros $(n=31)$, estudantes de Agrotécnica $(n=60)$ e trabalhadores da limpeza pública $(\mathrm{n}=51)$. A freqüência de positivos com anticorpos antileptospira, nestes grupos, foi de $46,7 \%(n=120)$. Nos demais grupos pesquisados ( 50 doadores de sangue, 51 visitas de pacientes do Hospital-Escola, 47 funcionários da indústria Fosfértil e 40 acadêmicos de Medicina) o grau de contanto com animais era proporcionalmente menor entre eles, a freqüência de pessoas com sorologia positiva foi de $19,3 \%(n=37)$. A difereça observada foi altamente significante $\left(\mathrm{x}^{2}=34,70 \mathrm{p}<0,00001\right)$, entre as freqüências de aglutininas antileptospira nos dois grupos, divididos conforme o grau de contato com animais. Os indivíduos com sorologia positiva tiveram, mais freqüentemente $(p<0,02)$, grau de contato maior. 
Comunicação. Tanares-Neto J, Andrade J, Hofer E, Oliveira GF, Couto-Jininior A. Freqüência de aglutininas para leptospira obsenadas em habitantes de Uberaba, Minas Gerais. Revista da Sociedade Brasileira de Medicina Tropical 29:55-58, jan-fev, 1996.

Na Tabela 1, o resultado da sorologia foi associado às variáveis pesquisadas. A freqüência de sorologia positiva foi maior nos indivíduos residentes na área rural (+ periferia) de Uberaba, sem alcançar significância estatística. Os naturais das áreas rurais foram, significantemente, mais positivos. A sorologia positiva contra leptospira foi maior, de foram altamente significativa, entre os indivíduos com ocupação nãoqualificada e menor renda familiar. Em acordo com a observação da associação indireta, de quanto maior foi a freqüencia de soropositivo menor foi o número de anos de estudo ( $p<0,0005)$. Assim, também esperarse-ia, o que foi observado, que os indivíduos classificados como mulatos ou negros tivessem sorologia positiva mais freqüentemente $(\mathrm{p}<$ $0,05)$.
Coerentemente, a freqüência do positivos, com aglutininas antileptospira, aumentou com a idade dos indivíduos $(\mathrm{p}<0,0001)$. Também a sorologia positiva foi maior no sexo feminino.

Muitos indivíduos tinham anticorpos para mais de um sorovar, com a seguinte distribuição, entre os $157 \mathrm{com}$ sorologia positiva:

$\begin{array}{crr}\text { n sorovars } & & \mathrm{n}(\%) \text { reagentes } \\ \begin{array}{c}1 \\ 2\end{array} & 39(63,1) \\ 3 & 12(7,6) \\ 4 & 5(3,2) \\ 5 & 3(1,9)\end{array}$

Tabela 1 - Associacào das variáueis pesquisadas e o resultado da sorologia para leptospirose, em inditiduos de vberaba, MG.

\begin{tabular}{|c|c|c|c|c|}
\hline \multirow[b]{2}{*}{ Variácel } & \multirow[b]{2}{*}{$\mathrm{n}$} & \multicolumn{3}{|c|}{ Anticorpos antileptospira } \\
\hline & & $\mathrm{n}$ & $\%$ & $\mathrm{x}^{2}(\mathrm{p})$ \\
\hline \multicolumn{5}{|l|}{ 1. Faixa etária (ano) } \\
\hline$<21$ & 83 & 18 & 21,2 & \\
\hline $211-130$ & 176 & 60 & 34,1 & \\
\hline $311-140$ & 91 & 29 & 31,9 & $31,46(<0,0001)$ \\
\hline $411-150$ & 53 & 20 & 37.7 & \\
\hline$>50$ & 42 & 30 & 71,4 & \\
\hline \multicolumn{5}{|l|}{ 2. Sexo } \\
\hline masculino & 328 & 107 & 32,6 & $3.86(<0,05)$ \\
\hline feminino & 117 & 50 & 42.7 & \\
\hline \multicolumn{5}{|l|}{ 3. Grupo racial } \\
\hline branco & 235 & 73 & 31,3 & $3,88(<0,05)$ \\
\hline negro $(+$ mulato $)$ & 210 & 84 & 40,0 & \\
\hline \multirow{2}{*}{\multicolumn{5}{|c|}{$\begin{array}{l}\text { 4. Residência atual } \\
\text { Uberaba }\end{array}$}} \\
\hline & & & & \\
\hline centro & 363 & 121 & 33.3 & $3.27(>0,05)$ \\
\hline periferia + rural & 82 & 36 & 43.9 & \\
\hline \multicolumn{5}{|l|}{ 5. Procedência } \\
\hline rural & 106 & 48 & 45.3 & $6.09(<0,01)$ \\
\hline urbana & 339 & 109 & 32,2 & \\
\hline \multicolumn{5}{|l|}{ 6. Anos de estudo } \\
\hline 0 & 22 & 10 & 45,4 & \\
\hline $11-14$ & 124 & 55 & 44,4 & \\
\hline $51-18$ & 116 & 50 & 43.1 & $24,69(<0,0005)$ \\
\hline $91-111$ & 85 & 26 & 30,6 & \\
\hline$>11$ & 98 & 16 & 16.3 & \\
\hline \multicolumn{5}{|l|}{ 7. Ocupacào atual } \\
\hline não-qualificacta & 137 & 55 & 40,1 & \\
\hline semicualificada & 173 & 71 & 41,0 & $12,90(<0,005)$ \\
\hline qualificada & 135 & 31 & 23,0 & \\
\hline \multicolumn{5}{|c|}{$\begin{array}{l}\text { 8. Renda familiar mensal } \\
\text { (cm salário mínimo) }\end{array}$} \\
\hline $11-13$ & 325 & 131 & 40.3 & \\
\hline $41+110$ & 60 & 15 & 25,0 & $13,93(<0,001)$ \\
\hline$>10$ & 60 & 11 & 18,3 & \\
\hline \multicolumn{5}{|c|}{ 9. Gratu de contato com animais } \\
\hline menor & 203 & 60 & 29.6 & $5.36(<0,02)$ \\
\hline maior & 242 & 97 & 40,1 & \\
\hline
\end{tabular}


Comunicação. Tavares-Neto J, Andrade J, Hofer E, Oliteira GF, Couto-Jünior A. Freqüencia de aglutininas para leptospira observadas em babitantes de Uberaba, Minas Gerais. Revista da Sociedade Brasileira de Medicina Tropical 29:55-58, jan-fev, 1996.

Dos 24 sorovars componentes da bateria de antígenos, somente castellonis e celledoni não mostraram presença de anticorpos para leptospira nos soros examinados. As respostas com aglutininas dirigidas contra os sorovars bataviae, copenhageni, hebdomadis e sejroe não foram monotípicas, mas ao mesmo tempo contra outros sorovars. Os sorovares de Leptospira mais freqüentemente detectados foram: patoc $(\mathrm{n}=88)$, panama $(\mathrm{n}=40)$, australis $(\mathrm{n}=28)$ e icterobaemorrbagiae $(\mathrm{n}=$ 28). As variáveis pesquisadas, listadas na Tabela 1, tiveram distribuição semelhante nos quatro sorovars mais freqüentes.

Estes resultados estão de acordo com os dados da literatura ${ }^{3} 10$ 12. A forte associação com as variáveis associadas às piores condições de vida, especialmente as sanitárias e sócio-econômicas, e contato com animais está coerente com o conhecimento epidemiológico da doença ${ }^{1}$.

No Brasil, a população negra, e os seus descendentes, apresentam piores indicadores sócio-econômicos, provavelmente disto decorreu a freqüencia maior da sorologia antileptospira entre os negros e mulatos estudados.

No ambiente domiciliar e peridomiciliar, os fatores associados à transmissão da leptospira estão presentes, ou mesmo em condições mais favoráveis, resultando em risco maior para o sexo feminino, por ter atividades ocupacionais mais vinculadas àqueles ambientes.

Os resultados evidenciaram também a importância da leptospirose humana em Uberaba, o que já havia sido alertado por Ribeiro e col $^{11}$ quando estudaram bovinos de município próximo. Os raros casos de leptospirose-doença podem ser devidos ao pouco conhecimento na formulação diagnóstica, na falta de apoio laboratorial, na freqüência elevada das formas assintomáticas ou atípicas ${ }^{1}$. Mas, principalmente, as condições sanitárias melhores do Triângulo Mineiro, quando comparadas com às de outras regiões brasileiras, bem como de outras condições ecológicas e sócio-econômicas desfavoráveis à transmissão da $L$. interrogans.

Este estudo demonstra também a importância e a necessidade da implantação de programa de vigilância epidemiológica, com busca ativa de casos, especialmente nos períodos chuvosos das regiôes com características sócio-econômicas semelhantes às do Triângulo Mineiro.

\section{REFERÊNCIAS BIBLIOGRÁFICAS}

1. Acha PN, Szyfres B. Leptospirosis. In: Acha PN, Szyfres B (eds) Zoonosis y enfermedades comunes al hombre y a los animales. Organizacion Panamericana de la Salud, $\mathrm{n}^{\circ}$ 503, Washington, $\mathrm{p}$. 112-119, 1986.

2. Alexander Ad. Serological diagnosis of leptospirosis. In: Rose NR, Friedman $\mathrm{H}$ (eds) Manual of clinical immunology, American Society for Microbiology, Washington, p. 542, 1980.

3. Andrade J, Brandão AP. Contribuição ao conhecimento da epidemiologia da leptospirose human em especial referência ao grande Rio, Brasil, no período de 1970 a 1982 . Memórias do Instituto Oswaldo Cruz 82:91-100, 1987.

4. Corrêa MOA. Human leptospirosis in Brazil. International Journal of Zoonoses 2:1-9, 1975.

5. Corrêa MOA, Mearim AB. Leptospirose no Brasil Levantamento bibliográfico de 1917 a 1970. Revista do Instituto Adofo Lutz 31:87-101, 1971.

6. Mearin AB, Corrêa MOA. Leptospirose no Brasil. Levantamento bibliográfico de 1971 a 1977. Revista do Instituto Adolfo Lutz 37:131-140, 1977.

7. Myers DM. Manual de métodos, para el diagnóstico de laboratório de la leptospirosis. Nota técnica $n^{\circ} 30$. Organização Panamericana de Saúde/Organização Mundial de Saúde, 1985.

8. Oliveira G, Couto Jr AS, Tavares-Neto J, Andrade J, Hofer E. Freqüencia de anticorpos antilisteria e antileptospira, em amostra de risco e controles, de Uberaba/MG. In: Resumos do XXVII Congresso da Sociedade Brasileira de Medicina Tropical 24(supl):162, 1991.

9. Organização Mundail de Saúde. Rapport d'un group d'experts de l'OMS. Problémes actuales des recherches sur la leptospirose. Organizacion Mundail de la Santé, $\mathbf{n}^{\circ}$ 380, 1967.

10. Pereira MM, Andrade J. Human leptospirosis in a shum area in the city of Rio de Janeiro, Brazil - A serological and epidemiological study. Memórias do Instituto Oswaldo Cruz 85:47-52, 1990.

11. Ribeiro SCA, Gouveia MAV, Silva PL, Oliveira PR, Barbosa FC, Mamed DO. Levantamento sorológico em dois surtos de leptospirose bovina, em Uberlândia, Triângulo MIneiro. Arquivo Brasileiro de Medicina Veterinária e Zootecnia 40:415-423, 1988. 
Comunicação. Tavares-Neto J, Andrade J, Hofer E, Oliveira GF, Couto-Jünior A. Freqüência de aglutininas para leptospira obsenadas em babitantes de Uberaba, Minas Gerais. Revista da Sociedade Brasileira de Medicina Tropical 29:55-58, jan-fev, 1996.

12. Szatalowicz FT, Griffin TP, Stunkard JA. The international dimensions of leptospirosis. Journal of the American Veterinary Medical Association 155:2122-2131, 1969.
13. Weiberg A, Weber DJ. Prefácio. $I n$ : Weinberg A, Weber DJ (eds) Clínicas de doenças infecciosas da América do Norte. Interlivros, Rio de Janeiro, p.XV, 1991. 